\title{
Los diseños verticales y la agricultura unidos para la producción de alimentos en los Módulos para Huertas Urbanas Verticales
}

\author{
Vertical designs and agriculture joined for food \\ production in the modules for urban vertical gardens.
}

\author{
Fritz Hammerling Navas Navarro¹ y Luz Mila Peña Torres² \\ IIngeniero Forestal. Especialista en Docencia Universitaria. \\ Magister en Ciencias de la Educación. ${ }^{2}$ Tecnóloga Agroforestal. \\ 1,2Universidad Nacional Abierta y a Distancia UNAD. Escuela de Ciencias Agrícolas, \\ Pecuarias y del Medio Ambiente ECAPMA. Zona Centro Bogotá. Zipaquirá. Colombia.
}

1fritznavas@yahoo.es, ${ }^{2}$ luzmil10@gmail.com

\section{Resumen}

Los Módulos para Huertas Urbanas Verticales (MHUV) nacen como un híbrido de los jardines verticales y la agricultura urbana, siendo reconocidos los primeros desde hace unos 2500 años, principalmente con los jardines colgantes en Babilonia, y la segunda como una práctica efectuada por más de 700 millones de personas en el mundo. Los beneficios que los MHUV brindan son múltiples, pero tal vez el más significativo es el hecho de consumir alimentos libres de químicos, no transgénicos, regados con agua potable y orgánicos $100 \%$. Se presenta un "módulo culinario y medicinal", que puede ser dispuesto en el área de la cocina, azoteas, terrazas, balcones o patios, donde se cultivaron especies como tomillo, menta, hierbabuena, perejil, toronjil y romero para ser aprovechadas mediante el uso de cortes de secciones de tallos u hojas al momento de las preparaciones de los platos. El módulo está formado por tres canastillas de plástico, totalmente reciclables y resistentes al deterioro; cada canastilla tiene cuatro hileras y una capacidad para catorce plántulas. Internamente están forradas con un geotextil negro; y luego son cubiertas con una malla (polisombra), la cual ayuda a sostener el sustrato
\end{abstract}

y las plántulas; cada canastilla descansa sobre una estructura en madera reciclada (de estibas o guacales) que además de sostenerla verticalmente, cumple la función de impermeabilizante. La medida estándar de cada canastilla es de $0,55 \mathrm{~m}$ x 0,33m x 0.14m. Cada módulo posee un equipamiento de mangueras para goteo, conectores para riego y un instructivo de uso. Se demuestran las ventajas de la Agricultura Urbana conjugadas con la belleza y dinamismo de los jardines verticales, lo que genera diseños útiles desde el punto de vista de producción de alimentos y ornato a los espacios donde los Huertos Urbanos Verticales sean dispuestos.

Palabras Clave: agricultura orgánica, agricultura urbana, autoconsumo, compost, jardines verticales

\section{Abstract}

Modules for Vertical Urban Gardens (MHUG) are a hybrid of vertical gardens and urban agriculture. Vertical gardens have been recognized for the past 2500 years, mainly in the form of the Hanging Gardens of Babylon, while urban agriculture is being practiced today by more than 700 million 
people worldwide. The benefits that MHUV offers are multiple, but perhaps the most significant is the consumption of foods free of chemicals, free of GMO's, irrigated with potable water, and that are $100 \%$ organic. It is presented a "culinary and medicinal module" that can be implemented in the kitchen area, on roofs, terraces, balconies or patios, where species such as thyme, mint, peppermint, parsley, lemon balm and rosemary can be at hand when preparing dishes. The module consists of three plastic baskets that are recyclable and resistant to decay. Each basket has four rows with space for fourteen seedlings. The baskets are first lined on the interior with a black geotextile, and then are covered with a mesh (polisombra) which helps support the substrate and seedlings. Each basket rests on a structure made of recycled wood (from pallets or crates) that both holds the basket vertically and serves as a rain cover. The cages measure $0.33 \mathrm{~m}$ by $0.55 \mathrm{~m}$ by $0.14 \mathrm{~m}$. Each module comes with hosing and connectors for a drip irrigation system, and an instructional manual. The modules demonstrate the benefits of urban agriculture combined with the beauty and modality of vertical gardens, leading to useful applications for food production and decoration in the spaces where vertical urban gardens are possible.

Key words: auto-consumption, compost, organic agriculture, urban agriculture, vertical gardens

\section{Introducción}

Los Módulos para Huertas Urbanas Verticales MHUV nacen como un híbrido de los jardines verticales y la agricultura urbana. La jardinería vertical es reconocida desde hace unos 2500 años, principalmente con los jardines colgantes en Babilonia, referenciados por Antípatro de Sidón en el siglo II a.C. al describirlos como una de las siete maravillas del mundo (National Geographic, 2011). Aunque son llamados colgantes, la verdad es que los griegos y los romanos al darle tal denominación, quisieron expresar que eran de varios pisos, construidos los unos sobre los otros. Cada uno de sus 20 pisos tenía una capa de tierra, suficiente para sostener toda clase de árboles (Saab, 1938).

En la actualidad no se pueden dejar de nombrar los murs vegetaux, paredes verdes, patentadas por el Botánico Francés Patrick Blanc, quien es uno de los principales exponentes de este arte. Blanc ha desarrollado más de 130 trabajos en sitios privados y de interés público, como en el parlamento de Bruselas, en el palacio presidencial de Tbilisi en Georgia y, actualmente, se encuentra trabajando en más de 10 proyectos alrededor del mundo (Blanc, 2012).
Según Blanc su inspiración creadora nace de la observación metódica de las plantas epífitas y su capacidad para sustentarse, incluso asidas a cables de luz; los diversos sistemas radiculares, preparados para soportar las plantas en las condiciones más extremas; los modelos naturales de arquitectura vegetal, el multicolor de las hojas de las especies rastreras y arbustivas; la adecuación fenotípica de los individuos instalados sobre zonas rocosas y la variedad de formas y colores de las flores de las plantas. Todo esto traído al paisaje de cemento y piedra que es la ciudad para romper ese gris y dar alternativas a la escasez de espacios verdes, labor en la cual converge la imaginación de arquitectos, paisajistas y botánicos (Fiarello, 2004).

Blanc no es el único exponente de este arte, desde 1930 el brasileño Roberto Burle Marx comenzó a crear arquitectura paisajística, donde combina sus conocimientos profesionales como artista plástico con el amor e interés por las plantas. Una frase pronunciada por Marx: "Quien no observa no vive realmente", demuestra su vinculación estética con el concepto de paisajismo, tomando como plataforma lo urbano, al igual que Falú y Marengo (2004), quienes aseguran que se está en el inicio 
del "milenio urbano", en el cual la ciudad ocupa un rol nuevo y central en el panorama mundial globalizado y, particularmente, en las situaciones de bloques supranacionales.

Los aglomerados urbanos hoy disputan espacios de liderazgo de distintas naturalezas (financieros, económicos, culturales), lo cual hace que las ciudades y sus gobiernos se constituyan en terreno fértil para impulsar cambios, a la vez que son el escenario en el que se expresan todas las contradicciones sociales.

\section{Desarrollo de la Agricultura Urbana}

En Bogotá, con el Proyecto de Acuerdo 386 se ha dado un paso firme hacia el paisajismo arquitectónico, determinando que los techos y muros verdes hoy en día son una tendencia a nivel mundial, máxime cuando fenómenos como el calentamiento global, la pérdida de la capa de ozono, el Niño y la Niña parecen traer consecuencias graves para nuestro planeta. Además de los beneficios estéticos por el impacto de lo verde y el aprovechamiento de espacios, los techos y muros verdes favorecen el ambiente y mejoran la calidad de vida de nuestros habitantes (Proyecto de Acuerdo No. 386 de 2009); siendo fundamentado en el Acuerdo 308 de Junio 9 de 2008, por el cual se adopta el Plan de Desarrollo Económico, Social, Ambiental y de Obras Públicas para Bogotá, D. C., 2008 - 2012 "Bogotá Positiva: Para Vivir Mejor". En este se promueve la existencia de una Política Distrital de seguridad alimentaria y nutricional que tiene como enfoque el derecho fundamental de los ciudadanos a la alimentación y al agua y que cuenta dentro de sus líneas de acción la promoción de prácticas de agricultura urbana.

Para hablar de Agricultura Urbana (AU) se presentan dos definiciones: el primer concepto dado por la FAO, (1999): "Pequeñas superficies (por ejemplo, solares, huertos, márgenes, terrazas, recipientes) situadas dentro de una ciudad y destinadas a la producción de cultivos y la cría de ganado menor o vacas lecheras para el consumo propio o para la venta en mercados de la vecindad". E segundo concepto dado por el Grupo Nacional de Agricultura Urbana en Cuba, 2003, (en González et al., 2008) es: "La producción de alimentos dentro del perímetro urbano y periurbano, aplicando métodos intensivos, teniendo en cuenta la interrelación hombre-cultivo-animal-medio ambiente y las facilidades de la infraestructura urbanística, que propician la estabilidad de la fuerza de trabajo y la producción diversificada de cultivos y animales durante todo el año, basadas en prácticas sostenibles que permiten el reciclaje de los desechos".

La AU que ha estado vinculada a temas como desarrollo sostenible, insuficiencia alimentaria, agricultura ecológica, educación medioambiental, calidad de vida, degradación ambiental, entre otros (Zaar, 2011), toca a las puertas de los hogares con el fin de tener una producción propia de plantas para el consumo y unirse a los más de 700 millones de residentes en las ciudades del mundo que son alimentados por estas siembras no rurales (ONU, 2005). No hay que pensar que esta población pertenece solamente a las clases pobres o a los países tercermundistas. Según el Food \& Fertilizer Technology Center, en Tokio para el año 2002 había cerca de 314 hectáreas sembradas en zonas urbanas y periurbanas (Tsubota, 2007) y según un informe del IPES (Instituto de promoción del desarrollo sostenible), Cuba cosechó en el primer trimestre de 2008 alrededor de 406.000 toneladas de productos por el sistema de AU, empleando más de 300.000 personas. Esto siguiendo los principios de la FAO (2005), donde se tienen en cuenta procesos participativos para la mejora de la calidad de vida de la población urbana y la gestión urbana social y ambiental sustentable de las ciudades.

Sin embargo, es evidente que los primeros beneficiados con la AU son los pobres, los desempleados y los niños. En su programa de acción integral de atención a poblaciones desarraigadas de la Agencia Presidencial de Cooperación Internacional de Colombia, (APC 2009) se logró 
impactar de forma directa a 4.300 personas de la localidad de Usme en Bogotá y de las comunas 3 y 8 en Medellín, considerados espacios sociales de población vulnerable y desplazada. Además asegura la APC que se generaron 490 empleos en 250 familias, aprovechando un área cultivada

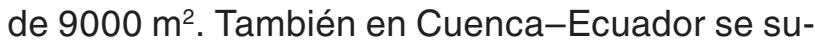
braya el rol social que la AU ha desarrollado, al involucrar ancianos jubilados, personas incapacitadas y revalorizando el papel de la mujer en la comunidad (Cosecha Urbana, 2004). También en Kano-Nigeria las granjas urbanas contribuyen de forma significativa a la nutrición, la seguridad alimentaria familiar, el empleo y el ambiente de la ciudad (Binns \& Lynch, 1998).

Teniendo en cuenta las consideraciones de Soriano (2006): "Una población puede considerarse segura alimentariamente cuando la comida está disponible en cualquier momento, cuando todas las personas tienen medios de acceso a ésta, cuando nutricionalmente sea adecuada en términos de calidad, cantidad, variedad y cuando sea aceptada en su contexto cultural". Los Módulos para Huertas Urbanas Verticales (MHUV) ofrecen a sus participantes la posibilidad de tener un acercamiento al autoabastecimiento y propender por la seguridad alimentaria de sus hogares, rompiendo con la cadena de distribuidores, que para Forero (2006), es asumida por una amplia gama de agentes: los productores que llevan directamente sus cosechas, los productores que se han consolidado como comerciantes, los intermediarios rurales que compran las cosechas de varios productores y las venden principalmente en Corabastos, transportadores que se encargan de llevar las cosechas de los productores mediante el cobro de fletes y, en ocasiones, de comisiones; comisionistas de los mayoristas de Corabastos y cooperativas que tienen por lo regular contratos con los supermercados. Agentes estos que en la mayoría de los casos incrementan exageradamente el precio de los alimentos que el consumidor final compra, teniendo como principal excusa el factor transporte, que para Colombia el costo del mismo es $20 \%$ superior al de Argentina, 38\% al de México y $13 \%$ al de Brasil (Cámara de Comercio de Bogotá, 2010).

Estos datos son relevantes, teniendo en cuenta que en Colombia según el periódico EL Heraldo (2012) existen 5,5 millones de personas sin empleo. Y según el diario El Eespectador (2012), para 2011 en Colombia había 21'510.000 pobres y pobres extremos, lo cual corresponde al $47,8 \%$ del total de la población en el país; convirtiendo a Colombia en uno de los países más pobres del mundo y de paso en uno de los primeros con mayor inequidad, ya que el $5 \%$ de las personas más ricas disponen de un tercio del ingreso total nacional; el 20\% intermedio de población se lleva el segundo tercio; y el $75 \%$ restante de la gente, los de menor ingreso, disponen del tercio restante (Chaves, 2011).

Son muchos los países en los cuales se desarrollan experiencias de agricultura urbana como respuesta social a los bajos niveles de seguridad alimentaria, principalmente en América Latina y el Caribe, donde la desnutrición crónica infantil alcanza el 13\%, agravado por un bajo consumo de frutas y hortalizas ubicado por debajo del estándar recomendado por la Organización Mundial de la Salud que es de 400 g/per cápita/día (Gobernación de Antioquia, 2009), y también por los elevados precios de algunos productos básicos, que gracias a la demanda de biocombustibles permiten el desabastecimiento de alimentos de la canasta familiar. Así Msangi y Rosegrant (2006) exponen que algunos formuladores de políticas y analistas expresan su preocupación por un crecimiento agresivo de la producción de biocombustibles que podría desplazar la producción de cultivos alimentarios en algunos países en desarrollo, y crear tensión entre la necesidad de generar energía y la necesidad de producir alimentos para consumo humano y animal. El escenario de "crecimiento agresivo de biocombustibles" describe importantes aumentos en los precios mundiales de los cultivos de materia prima para la producción de biocombustible, como son: yuca, maíz, oleaginosas, remolacha azucarera, caña de azúcar y trigo. 
Para Millán y Granados (2006), la baja calidad de los alimentos y la inequidad en el suministro de los mismos por problemas de acceso económico, generan un estado nutricional deficiente en la población de bajos ingresos, particularmente, en la población infantil, que necesita una taza al día de vegetales ricos en vitaminas para satisfacer sus requerimientos de micro nutrientes, situación ante la cual la producción urbana ha entrado a ofrecer una solución parcial. Según la OMS (2002, citado por Vega, 2006), la carencia de hierro afecta a más de 2000 millones de personas en el mundo. Los más gravemente afectados son los niños pequeños y las mujeres en edad fértil porque son los que más requieren el hierro para el crecimiento y la gestación. Sin embargo, una forma de disminuir este daño es con el consumo periódico de hortalizas, especialmente espinaca (Spinacia oleracea) y acelga (Beta vulgaris), que con la producción en la AU se alcanzan tasas de 10 a 12 kilogramos cosechados por semana teniendo un espacio de cultivo de $30 \mathrm{~m}^{2}$ (Chica, 2004).

La AU para los bogotanos ha sido más una especie de salvavidas alimentario, llevada a cabo en momentos de alta necesidad, que una experiencia de mitigación agroecológica, como lo señala Granados (2007). La agricultura urbana en Bogotá ha acompañado algunos de los procesos de urbanización de los últimos 35 años como aquel que promovieron campesinos en los 60 y que trajo consigo la ocupación al sur oriente contra los cerros orientales; estos campesinos promovieron la formación de barrios como Juan Rey, La Gloria, Santa Cecilia en donde los cultivos de papa y maíz legitimaban la posesión de los nuevos predios. En la década de 1980 la cuenca del río Tunjuelo se llenó de habitantes del centro que no soportaron los nuevos ajustes a los impuestos promovidos en aras de sostener la emergente industria bogotana. La lucha sindical trajo la luz, y las huertas familiares constituyeron la fuente de alimento para los habitantes de los nuevos sectores de Ciudad Bolívar y Bosa.

El concepto de urbanización se trabajó desde lo señalado por Martínez (1992), "La urbanización ocurre por la emigración rural a causa de la pérdida de población activa del sector agrícola al aumentar su productividad (medida esa productividad no con un criterio ecológico, sino con un criterio económico convencional) y también aumenta el tamaño de las ciudades por el crecimiento de la propia población ya urbanizada".

Planeta Paz (2006), en la presentación de su documento Bogotá: Autonomía Agroalimentaria, asegura que para las personas pobres "La demanda por alimentos tiene una elasticidad muy alta", lo cual significa que a la menor fluctuación de los precios de productos agrícolas, se genera una drástica disminución de las cantidades de alimentos que las personas más pobres demandan, lo que aumenta dramáticamente los índices de familias subalimentadas. El mismo informe, en contraste, afirma que una mínima disminución de los precios, hace que se aumente visiblemente el consumo de alimentos por este mismo grupo de familias.

A diferencia de los cultivos tradicionales, donde se realizan fertilizaciones continuas con agroquímicos y aspersiones con fungicidas, en la $\mathrm{AU}$ se puede prescindir de estos, ya que se puede usar como sustrato el compost y el fungicida no se hace indispensable debido a la extensión reducida de los cultivos. Esto, además de ser un ahorro económico, también lo es ambiental y se toma como ejemplo el caso del Fósforo como nutriente esencial de todas las plantas y animales y que junto con el Nitrógeno y el Potasio son los componentes básicos para la fertilización agrícola.

Según Cofie (2010), el Fósforo se hace crucial para el sistema mundial de abastecimiento de alimentos, y las reservas en los EE.UU., uno de los 5 países con este mineral, han ido disminuyendo rápidamente desde 2006 y se predice que las mismas serán consumidas en unos 25 años. Y si se observan los cálculos de Drechsel (2010), donde se indica que entre el $65-80 \%$ del Nitrógeno y Fósforo, respectivamente, se pierde a través de fosas sépticas, vía aguas residuales o residuos sólidos dispersos en el ambiente, se hace más relevante la participación de la AU a nivel global, con el 
aprovechamiento de residuos orgánicos producidos en hogares y comunidades.

Algunas estadísticas indican que en un cultivo tradicional de lechuga (Lactuca sativa) se requiere como media $95 \mathrm{~kg}$ de Nitrógeno, $200 \mathrm{~kg}$ de Potasio y $120 \mathrm{~kg}$ de Fósforo. Estos fertilizantes aumentan la contaminación directa de fuentes de agua, que se inicia en los mismos procesos de fabricación, lo que genera problemas ambientales graves a los suelos, aire y agua, así como a las personas que directa e indirectamente se relacionan con estas labores. El efecto más nocivo recae en los consumidores quienes finalmente son los que ingieren estos productos. Según un artículo publicado por la Universidad Nacional de Colombia (González y Mejía, 1995), se evidencia la presencia de concentraciones significativas de metales como Mercurio $\mathrm{Hg}$, Plomo Pb, Cadmio $\mathrm{Cd}$, Cromo $\mathrm{Cr}$, entre otros, en sectores de la cuenca del río Bogotá; los cuales a corto y mediano plazo causan problemas respiratorios, gastrointestinales, cardiovasculares y nerviosos.

Por el contrario, en un artículo de Arce y Peña (2010), se describe cómo en la ciudad de Bogotá se experimentaron diversos sustratos sin fertilizantes en el cultivo de lechuga (Lactuca sativa), encontrando que las mezclas de humus sólido (combinado con suelo y cascarilla cruda) y compost (combinado con cal y cascarilla cruda) tenían mejores características en términos de tasa de germinación, capacidad de retención de la humedad, infiltración y drenaje, color de la cosecha, conservación ambiental, coste y disponibilidad a nivel local, y calidad. Ademas, que la producción de lechuga utilizando estas mezclas consiguió resultados similares a los obtenidos con el uso del sustrato comercial. Esta investigación se desarrolló en planteles escolares de diversas zonas de la ciudad, mediante la participación de hogares, estudiantes y profesores en las labores de Agricultura Urbana.

Según Jianming y Wenhua (2008), en Pekín se siguen buscando alternativas en la AU para la utilización de fuentes de consumo de agua como el reúso de aguas residuales, la cosecha de aguas de lluvia y la mejora del manejo del agua. Ejemplos que denotan la importancia de los pequeños y medianos proyectos alrededor de la Agricultura Urbana.

Otro concepto que se debe involucrar en el desarrollo de la AU es el de bienestar, no sólo alimentario y económico, sino también el bienestar emocional y medicinal; como reza un proverbio chino: Si quieres ser feliz una hora, emborráchate. Si quieres ser feliz un día, mata al cerdo.

Si quieres ser feliz una semana, haz un viaje. Si quieres ser feliz un año, cásate. Si quieres ser feliz toda la vida, ten un huerto (Winkels, 2010). En Japón, por ejemplo, las empresas han venido creando jardines urbanos en las azoteas y sótanos, donde los empleados pasan parte de su descanso efectuando prácticas agrícolas y de cuidado de los cultivos, lo que permite la relajación y reducción del estrés laboral.

En Alemania buscan en la horticultura urbana ya no un mecanismo de supervivencia como en el pasado, pero sí de recreación y descanso esencial para el equilibrio psíquico de los que intervienen en estos proyectos (Sánchez, 2007). Según Cantor (2010) la AU se efectúa en la ciudad de Bogotá tejiendo redes cotidianas entre vecinos, revalorando las capacidades y conocimientos del campo, creando un espacio con valor paisajístico, generando alimentos sin tener que dar dinero por ellos, enmarcándose en una idea de bienestar colectivo que trasciende lo monetario. El valor de la huerta es que representa un espacio tranquilo, autónomo y de resistencia al medio hostil que significa la ciudad.

La AU también ha intervenido en el fomento de la medicina casera, con el cultivo de plantas aromáticas, medicinales y productos derivados como infusiones, extractos y esencias en los hogares, con lo cual se podría pensar en reducir el gasto que los hogares pobres efectúan en salud y medicina, que según el Instituto de Promoción del Desarrollo Sostenible IPES (2002), el calculado para América 
Latina es del $15 \%$ del sueldo mensual. Dentro de las especies aromáticas que el Proyecto RESA (2008) recomienda para sembrar en clima frío están la Manzanilla (Matricaria chamomilla) y el Llantén (Plantago major), y dentro de los beneficios que expone para la AU está principalmente el de las plantas medicinales en casa que mejoran nuestra salud y la salud del territorio en general. La siembra ayuda a relajarse a tener un paisaje más agradable y a ocupar el tiempo libre.

En Pereira, se brinda un buen ejemplo en la comunidad de la Comuna Ferrocarril, quienes a partir de una serie de lotes liberados por condición de riesgo han desarrollado actividades de siembra para diversos usos, reivindicando especies agrícolas olvidadas y promoviendo la agricultura como una práctica cultural para el buen uso del tiempo libre. Se tienen cuatro tipos de plantas en sus cultivos: las medicinales, con 22 especies; las plantas alimenticias, con 7 especies; las plantas mágicas, con 6 especies y las plantas ornamentales, con 6 especies. Son aprovechadas todas por la comunidad para sus necesidades y requerimientos (UTPUniversidad Tecnológica de Pereira, 2011).

En Bogotá la experiencia más relevante en tiempo y participación es la efectuada por el Jardín Botánico José Celestino Mutis, la Universidad del Rosario y el IPES - Promoción del Desarrollo Sostenible, que según informe del 2008, ha capacitado a 41.245 personas, quienes han participado en 149 núcleos en la ciudad; han cultivado alrededor de 60 diferentes especies en zonas duras, con un promedio en tamaño de $23 \mathrm{~m}^{2}$ (patios, balcones, azoteas, etc.), zonas blandas, con un promedio en tamaño de $43 \mathrm{~m}^{2}$ (Jardines, antejardines, patios y lotes) y zonas mixtas (mezclando las dos anteriores) (Alcaldía, 2008). Con esta experiencia, posteriormente se publicó el Manual de Tecnologías de la $\mathrm{AU}$, donde se enumeran los ejes estratégicos y líneas de acción, promoción del conocimiento y consumo de especies vegetales andinas; capacitación y formación para mejorar hábitos alimenticios hacia un mejoramiento nutricional; aporte de alimentos a la canasta familiar (con la producción de especies frutales, hortalizas, aromáticas y medicinales) (Alacandía, 2009).

\section{Características de los Módulos para Huertas Urbanas Verticales MHUV}

Los MHUV adoptan las prácticas limpias y de autoconsumo de la Agricultura Urbana y las combinan con la intensión estética y de ahorro de espacio de los jardines verticales. En un estudio experimental se utilizó un "módulo culinario-medicinal. En estos módulos se cultivan especies como Tomillo (Thymus vulgaris L.), Menta (Mentha piperita), Hierbabuena (Mentha sativa), Perejil (Petroselinum crispum), Toronjil (Melissa oficinalis) y Romero (Rosmarinus officinalis) para ser aprovechadas mediante el corte seleccionado de tallos $u$ hojas al momento de las preparaciones de los platos (Gómez, 2010).

Los huertos urbanos se disponen casi en cualquier recipiente que pueda soportar una cantidad de tierra mínima para el sustento de la especie que se desea plantar. En Bogotá uno de los diseños verticales es el contenedor tubular, el cual es ideal para espacios limitados, pero este debe tener una estructura metálica fija para soportar el peso del cultivo; mientras que los MHUV no necesitan estructura metálica y las rodachinas le dan movilidad en interiores o exteriores. El contenedor tubular es una bolsa plástica negra, gruesa, de $1.00 \mathrm{~m}$ de largo x $0.26 \mathrm{~m}$ de ancho, en cuyo interior se dispone el sistema de riego y el sustrato necesario para la siembra y desarrollo de diversas hortalizas y frutales pequeños (IPES, 2008).

Un módulo completo está formado por tres canastillas de plástico, una estructura (de $0.11 \mathrm{~m} \times 0.60$ $\mathrm{m}$ ) en madera reciclada y un sistema de riego por goteo; sistema que posee las siguientes ventajas: reduce las pérdidas directas por evaporación, no causa humedecimiento del follaje, aumenta el rendimiento por unidad de agua aplicada, mejora la aireación, aumenta la provisión de nutrimentos disponibles, reduce el desarrollo de insectos y de enfermedades y ayuda a controlar la erosión (Goyal et al., 2006). 
Cada canastilla tiene cuatro hileras y una capacidad para catorce plántulas. Internamente son forradas con un geotextil negro para evitar pérdida de humedad, que aunque presenta un costo alto, reduce significativamente el impacto ambiental y favorece el uso de materiales reciclados (Giménez, 2010), y luego son cubiertas con una malla (polisombra), la cual ayuda a sostener el sustrato y las plántulas; la medida estándar de cada canastilla es de 0,55 m x 0,33 $\mathrm{m} \times 0.14 \mathrm{~m}$.

El sistema de siembra, que según Aparicio (2010), en evaluaciones hechas con Cilantro (Coriandrum sativum), Cebollino (Alliu fistolosum) y Repollo (Brassica oleracea var. Capitata), presentó los mayores beneficios en una misma área de terreno, generó ingresos adicionales, sustentando 14 plántulas (en triángulo o tresbolillo), seleccionadas entre hortalizas, hojas verdes, hierbas medicinales o aromáticas, dependiendo del gusto o necesidades del cultivador. Como ejemplo se implementó un módulo vertical de plantas medicinales y aromáticas instalado en la cocina, para cosechar hojas al momento de requerirlas en la práctica culinaria.

Siguiendo las recomendaciones del Jardín Botánico de Bogotá (2007), las canastillas se llenaron con sustrato (compost, cascarilla y tierra), ya que los elementos mezclados ofrecen mejores características que utilizados por separado. La preparación del sustrato, siembra y mantenimiento (fertilizantes) de las plántulas tiene un manejo libre de químicos; permitiendo que los interesados en estos MHUV efectúen el proceso de producción de abonos y fertilizantes orgánicos (humus o compost), aprovechando la mayor parte de los residuos producidos en los hogares. Esto ayuda a la sostenibilidad ambiental al no utilizar fertilizantes químicos para el desarrollo y mantenimiento de las plantas.

Según Peña (2007) el humus de lombriz influye en forma efectiva en la germinación de las semillas y en el desarrollo de las plantas y previene enfermedades. Se puede usar sin inconvenientes en estado puro y se encuentra libre de nemátodos.
Una de las características más importantes del humus de lombriz es su carga biológica, marcada por su elevado contenido de microorganismos y actividad enzimática.

Las tres canastillas, que forman un módulo, descansan sobre una estructura en madera reciclada (de estibas o guacales) que además de sostenerla verticalmente, cumplen la función de impermeabilizante, evitando fugas de humedad a las paredes cercanas donde podría instalarse el sistema. Esta estructura tiene una base con cuatro rodachinas para ofrecer la posibilidad de desplazamiento dentro del hogar.

El riego se efectúa por goteo, para permitir el ahorro de agua y para evitar desperdicios en los espacios donde se instale. Cada módulo posee un equipamiento de mangueras para goteo, conectores para riego y un instructivo de uso.

La disposición, forma y equipamiento de los MHUV es versátil y encaja en cualquier espacio, con posibilidad de reutilizarlo en caso de cambiar la especie por cultivar. Los MHUV pueden ser usados con el propósito de decorar espacios o como alternativa doméstica que colabore a suplir necesidades tanto económicas como alimenticias del consumidor. Esta propuesta resulta atractiva por el bajo costo en materiales, la facilidad que presenta su elaboración y el beneficio que logra proporcionar una dieta saludable.

\section{Beneficios de los Módulos para Huertas Urbanas Verticales MHUV}

Con los estudios de los Módulos de Huertos Urbanos Verticales (MHUV) se ha demostrado que:

- El diseño se presenta novedoso al tomar la belleza de la jardinería vertical y combinarla con la utilidad y necesidad de la agricultura urbana; siendo llamativo para cualquier persona y fácil de ubicar en cualquier espacio del hogar, oficina, restaurante $u$ otros lugares que acojan este sistema de producción alimentaria. 
- La sencillez y la practicidad es una de las características de estos módulos ya que no requieren de mucho dinero, los materiales usados son de fácil acceso y la mano de obra es mínima.

- Al comparar los beneficios ambientales, económicos, sociales y culturales que ofrecen los MHUV versus la producción agrícola tradicional; se observa la reducción en insumos agroquímicos, las ventajas económicas para el cultivador al no pagar por intermediarios ni transporte de productos alimenticios, el consumir alimentos sanos y libres de transgénicos o químicos. Esto en la medida que las semillas y el fertilizante son suministrados desde la planta de producción.
- Las variables del concepto de Calidad de Vida versus las Huertas Urbanas ofrecen resultados positivos sobre el bienestar, condiciones ambientales, generación de empleo, seguridad alimentaria, crecimiento económico y participación de la sociedad. Alguacil (2000) argumenta que en principio las necesidades se presentan por urgencias vitales en la condición del hombre, marco en el que se inscriben las relacionadas con la salud y la seguridad, las fisiológicas, las relaciones y los derechos sociales, pero también pueden surgir a partir de deseos esporádicos alentados por el Estado y el mercado (Figura 2).

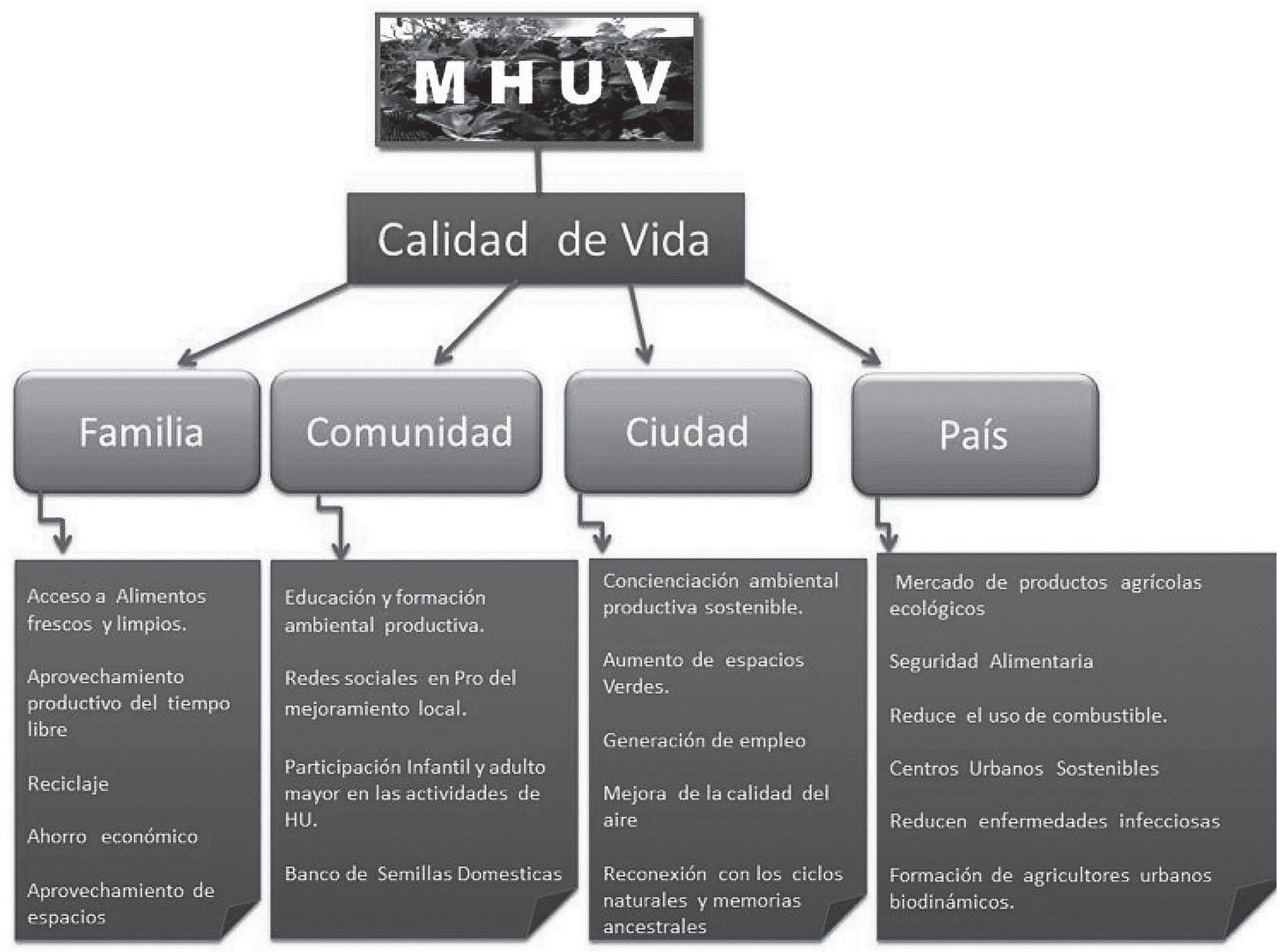

Figura 2. Relación calidad de vida y Huertas Urbanas. Fuente: Elaborado a partir de Alguacil (2000). 
- Es fácil desarrollar agricultura orgánica por medio de este sistema de plantación hecho en casa. En el modelo implementado en la cocina se observó la practicidad e higiene al cocinar y utilizar hierbas limpias y cultivadas por la misma persona.

- Se observa una buena adaptación de la hierbabuena, el perejil y el romero, mientras la albahaca y el tomillo no presentan buen desarrollo inicial. Es necesario realizar más pruebas para determinar si estas especies no se acoplan bien al sistema.

- En la fabricación de un módulo se recuperan aproximadamente $0,2 \mathrm{~m}^{2}$ de madera, lo que evita que las estibas con que se construye sean quemadas o enviadas al botadero y beneficia directamente el ambiente al disminuir la cantidad de $\mathrm{CO}_{2}$ dirigido a la atmósfera.

\section{Conclusiones}

Desde hace varios años muchos países, ciudades, embajadas, instituciones, las ONG y personas del común han venido incentivando y desarrollando proyectos de agricultura urbana en pos de buscar solución a la crisis alimentaria. Pero en esta búsqueda se ha demostrado que la agricultura urbana también es una fuente de relajación al brindar momentos de ocio con las plantas, beneficiando la salud psíquica, al igual que la salud corporal cuando son llevados a la mesa alimentos que no contienen trazas de químicos ni contaminantes.

Este trabajo demostró las ventajas de la Agricultura Urbana conjugadas con la belleza y dinamismo de los jardines verticales; se generan diseños útiles desde el punto de vista de producción de alimentos y ornato a los espacios donde los Huertos Urbanos Verticales sean dispuestos.

\section{Literatura citada}

1. Alcaldía Mayor de Bogotá. (2008). Agricultura Urbana en Bogotá: situación, perspectivas y retos. Bogotá: Inprenta Nacional.

2. Alcaldía Mayor de Bogotá. (200). . Agricultura Urbana, Manual de Tecnologías. Bogotá, Imprenta N.Alguacil, Julio. (2000). Calidad de Vida y Praxis Urbana. Madrid: Ed. Centro de Investigaciones Sociológicas.

3. Aparicio, R. (2010). Evaluación de dos Arreglos y dos Sistemas de Cultivo en la Producción de Repollo (Brassica oleracea var. Capitata), en Asocio con Cebollino (Alliu fistolosum) y Cilantro (Coriandrum sativum), con la Aplicación del Método del Cultivo Biointensivo de Alimentos. (Tesis de Pregrado). Universidad de El Salvador, Facultad de Ciencias Agronómicas, Departamento de Fitotecnia. San Salvador.

4. APC, Agencia Presidencial de Cooperación Internacional de Colombia. Acción Integral de Atención a Poblaciones Desarraigadas. Disponible en: : http://www. accionsocial.gov.co/BuenasPracticas/FichaResumen. aspx?id=238.2009

5. Arce, B. \& Peña, A. (2010) Sustratos comerciales para la Agricultura Urbana en Bogotá. Revista Agricultura Urbana. No. 23, 29.

6. Binns, T. \& Lynch, K. (1998 ). Feeding Africa's growing cities into the 21st century:the potential of urban agriculture. Journal of International Development. Journal of International Development. Volumen 10 ( 6), 777-793.

7. Blanc, Patrick. Sitio web: Vertical Garden Patrick Blanc. Disponible en línea: http://www.verticalgardenpatrickblanc.com Consultado Agosto de 2012

8. Cámara de Comercio de Bogotá. Plan Económico para la Competitividad del

9. Bogotá, Concejo de Bogotá. (2008). Acuerso 308 del 9 de junio de 2008. Por el cual se adopta el Plan de Desarrollo Económico, Social, Ambiental y de Obras Públicas para Bogotá, D. C., 2008 - 2012, Bogotá 'Positiva: Para Vivir Mejor.

10. Cantor, K. (2010). Agricultura urbana: elementos valorativos sobre su sostenibilidad. Cuadernos de Desarrollo Rural 7, (65), 61-87

11. Chaves, E. (2001). Colombia y sus pobrezas: las líneas de un debate. 16 de Octubre de 2011. Disponible en: http://www.rebelion.org/noticia.php?id=137515

12. Chica, C. (2004). Experiencia exitosa en la lucha contra el hambre de familias en situación de desplazamiento y desarraigo. Revista Trimestral Futuros No. 6. Volumen 2, 1-2. Disponible en: http://www.revistafuturos.info/futuros_6/caqueta_1.htm

13. Cofie, O., Van Veenhuizen, R., De Vreede, V. \& Maessen, S. (2010). Gestión de Residuos para la Recuperación de Nutrientes: Opciones y Desafíos para la Agricultura Urbana. Revista Agricultura Urbana. No. 23, 3. 
14. Cosecha Urbana. (2004). Memoria y Declaración: "La Integración de la Agricultura Urbana en el Desarrollo Sostenible de las Municipalidades". Centro Internacional de la Papa.

15. Drechsel, P., Cofie, O. \&, Danso, G. (2010). Cerrando los Ciclos Rurales - Urbanos de los Alimentos y los Nutrientes en África Occidental: Un Baño de Realidad. Revista Agricultura Urbana. No. 23, 8.

16. EL Espectador. (2012, 21 de Mayo). Editorial, Sobre la Pobreza. El Espectador. Disponible en:http://www.elespectador.com/opinion/editorial/articulo-347737-sobre- pobreza

17. El Heraldo. (2012, 29 de Febrero). Desempleo en Colombia se ubicó en el $12,5 \%$ en enero. El Heraldo. Disponible em: http://www.elheraldo.co/noticias/nacional/ desempleo-en-colombia-se-ubico-en-el-125-en-enero-58605.

18. Falú, A. \& Marengo, C. (2004). "Las políticas urbanas: desafíos y contradicciones". En Torres, Ribeiro, Ana Clara (compilador), El rostro urbano de América Latina, Buenos Aires, CLACSO, pp. 211-225.

19. FAO. (1999). Agricultura Urbana: algunas reflexiones sobre su origen e importancia actual. Disponible en: http://www.ub.edu/geocrit/b3w-944.htm

20. FAO (Junio 3 de 2005). Agricultura urbana impulsa seguridad alimentaria", Disponible en:

21. http://www.un.org/spanish/News/fullstorynews.asp?n ews $\mid \mathrm{D}=4714 \&$ criteria1=agricultura\&criteria2 Fiarello, Francesco. (2004). La arquitectura de los Jardines: de la antigüedad del siglo XX n p. 43.

22. Forero, A. (2006), El sistema de abastecimiento alimentario de Bogotá, análisis y propuestas. Bogotá: autonomía agroalimentaria. Diálogos y controversias. Capítulo 3. Primera edición. Bogotá, PUJ.

23. Giménez, A. ( 2010). Utilización de Agrotextiles. Análisis de viabilidad y modelización de la eficacia. (Tesis doctoral), Valencia: Universidad Politécnica de Valencia

24. Gobernación de Antioquia. (13 de octubre de 2009). Declaración de Medellín. Segundo Seminario Taller Internacional Agricultura urbana: Una herramienta para la Seguridad Alimentaria y la Lucha contra el Hambre. Medellín.

25. Gómez, B. (2010). Potencial Agroecológico de los Huertos Familiares en el Municipio de H. Cárdenas, Tabasco: Permanencia y Prospectiva de Desarrollo. (Tesis de Maestría). Montecillo, Texcoco, Estado de México. Colegio de Postgraduados.

26. González, M., Castellanos, A. \& Price, J. (2008). Testimonios: agricultura urbana en ciudad de la Habana. ACTAF: Asociación Cubana de Técnicos Agrícolas y Forestales.Editorial CIDISAV.

27. González, S. \& Mejía, L. (1995). Universidad Nacional de Colombia. Sede Bogotá.

28. Dirección Nacional de Servicios Académicos Virtuales. (s. f.) "Contaminación con Cadmio y Arsénico en suelos y hortalizas de un sector de la cuenca del río Bogotá" Tomado de La Revista de Suelos Ecuatoriales.
Disponible en: http://www.virtual.unal.edu.co/cursos/ ciencias/2000088/lecciones/Foros/ContaCadmio. html

29. Granados, S. (2007). Retos de la Gestión Ambiental en el establecimiento de Huertos Urbanos Domésticos y comunitarios [tesis de maestría], Bogotá, Pontificia Universidad Javeriana, Maestría en Gestión Ambiental.

30. Goyal, M., Martínez, M., Rivera, L., \& Rojas, N. (2006). Principios de Riego por Goteo. Capítulo V del libro Manejo de Riego Por Goteo. Autor : Dr. Megh R. GOYAL. Mayagüez, Puerto Rico.

31. IPES julio de 2009). Promoción del Desarrollo Sostenible. Huertos Orgánicos Urbanos en Azoteas, Terrazas y Patios Traseros. Serie Guías Prácticas ¿Cómo Hacerlo? Primera Edición. Lima Perú.

32. IPES (DICIEMBRE DE 2002). Lineamientos para la Formulación de Políticas Municipales para la Agricultura Urbana. Agricultura Urbana y Participación Ciudadana. Primera edición. Lima Perú.

33. Jardín Botánico de Bogotá. (Noviembre de 2007). Cartillas técnicas. Agricultura Urbana. Bogotá.

34. Jianming, C. \& Wenhua, J. (2008). Adaptándose a la Escasez de Agua: Mejorando fuentes de agua y su uso para la agricultura urbana en Pekín. Revista Agricultura Urbana. No. 20,. Pg. 11.

35. Martínez, J. (1992). De la economía ecológica al ecologismo popular. Editorial Icaria. Barcelona.

36. Msangi, S. \& Rosegrant, M. et al. (2006) Bioenergía y Agricultura: Promesas y Retos. Enfoque 14. Resumen 3 De 12. Diciembre de 2006. Disponible en: http://www. ifpri.org/sites/default/files/publications/focus14sp.pdf

37. Millán, J. \& Granados, S. (junio de 2006). Evaluación ecológica, agronómica y sociocultural de la agricultura urbana en el sector de Potosí. Capítulo 4. Primera edición. Bogotá.

38. Natinal Geographic. (Marzo de 2011). Revista. Las Siete Maravillas de la Antigüedad. No. 87. P. 50.

39. ONU. Organización de las Naciones Unidas. (2005). "FAO: Agricultura urbana impulsa seguridad alimentaria", Publicación en internet: Centro de Noticias ONU, Junio 3 de 2005. Disponible en: http://www.fao.org/ newsroom/es/news/2005/102877/index.html

40. Planeta Paz. (junio de 2006). Bogotá: autonomía agroalimentaria. Diálogos y controversias. Capítulo 1, Presentación. Primera edición. Bogotá.

41. Peña, E. (2007). El Humus de Lombriz: su generalización en la producción orgánica de posturas en cepellón para la Agricultura Urbana. Revista Agricultura Urbana. No. 3, 42-43. Disponible en: http://ftp.censa.edu.cu/ict/ Revistas\%20Cientificas\%20Cubanas/Agricultura\%20 Organica\%20\%28ACTAF\%29/Rev\%202007-3/19-HumusAU.pdf

42. Proyecto de acuerdo No. 386 de 2009. El Cual Se Implementan, Promueven y Estimulan Las Tecnologías de Creación de Techos Verdes en Bogotá, D. C. y se dictan otras disposiciones. Disponible en: http://www.alcaldiabogota.gov.co/sisjur/normas/Norma1.jsp?i=37533 
43. RESA. Proyecto Red de Seguridad Alimentaria. (2008). Agricultura Urbana en Bogotá. Proyecto Piloto en Ciudad Bolívar. Producimos Alimentos Sanos en Casa. Disponible en: http://www.humboldt.org.co/jardinesdecolombia/agricultura urbana/ Saab, Antonio. (1938). Jardines colgantes de Babilonia. Revista Pan, p.13. Bogotá.

44. Sánchez. L. (2007). Universidad el Bosque. La forma Urbana de la Agricultura. Revista de diseño Más D. Edición No. 1. Artículo on line disponible en: http://masd.unbosque.edu.co/content/edicion-01.

45. Soriano, R. (2006). Agricultura urbana en la ciudad de México: situación y perspectivas.

46. Revista Agraria nueva época. Disponible en: http://pupabiologos.tumblr.com/

47. Tsubota, K. (s. f.). "Urban Agriculture in Asia: Lessons from Japanese Experience". Food \& Fertilizer Technology Center, for the Asian and pacific Region. Disponible en: http://www.agnet.org/library/eb/576/
48. UTP - Universidad Tecnológica de Pereira. (2011) Instituto de Investigaciones Ambientales. Agricultura Urbana en espacios liberados. Estrategia socio-ambiental para fomentar la seguridad y soberanía alimentaria en la Comuna Ferrocarril de Pereira, Colombia.

49. Vega, R. (2006). Alimentación, Nutrición y Salud. Bogotá: autonomía agroalimentaria. Diálogos y controversias. Capítulo 3. Primera edición. Bogotá.

50. Winkels E. (2010, 6 de septiembre). Tener un huerto para ser feliz. El Periódico De Catalunya. Recuperado de: http://www.elperiodico.com/es/noticias/barcelona/20100906/tener-huerto-para-ser-feliz/468351.shtml

51. Zaar, M. Agricultura urbana: algunas reflexiones sobre su origen e importancia actual. En: Revista bibliográfica de Geografía y Ciencias Sociales, Universidad de Barcelona. ISSN: 1138-9796. Vol. XVI, № 944, 15 de octubre de 2011. Serie documental de Geo Crítica. Cuadernos Críticos de Geografía Humana. Disponible en: http:// www.ub.edu/geocrit/b3w-944.htm 